\title{
Dirichlet Brownian Motions
}

\author{
Hafedh Faires \\ Department of Mathematics and Statistics, Al Imam Mohammad Ibn Saud Islamic University (IMSIU), Riyadh, \\ KSA \\ Email: hmfaires@imamu.edu.sa, hafedh.faires@hotmail.com
}

Received 14 September 2014; revised 12 October 2014; accepted 6 November 2014

Copyright (C 2014 by author and Scientific Research Publishing Inc.

This work is licensed under the Creative Commons Attribution International License (CC BY).

http://creativecommons.org/licenses/by/4.0/

c) (†) Open Access

\begin{abstract}
In this work we introduce a Brownian motion in random environment which is a Brownian constructions by an exchangeable sequence based on Dirichlet processes samples. We next compute a stochastic calculus and an estimation of the parameters is computed in order to classify a functional data.
\end{abstract}

\section{Keywords}

Bayesian Model, Brownian Motion, Exchangeability, Gaussian Mixtures

\section{Introduction}

The Brownian motion is a very interesting tool for both theoretical and applied math. Brownian motion is among the simplest of the continuous-time stochastic processes, and it is a limit of both simpler and more complicated stochastic processes. In this paper we construct a new process called Dirichlet brownian motion by the usual i.i.d. Gaussian sequence used in Brownian motion constructions is replaced by an exchangeable sequence.

Despite its recent introduction to the literature, hierarchical models with a Dirichlet prior, shortly Dirichlet hierarchical models, were used in probabilistic classification applied to various fields such as biology [1], astronomy [2] or text mining [3] and finance [4]-[6]. Actually, these models can be seen as complex mixtures of real Gaussian distributions fitted to non-temporal data.

The aim of this paper is to extend these models and estimate their parameters in order to deal with temporal data following a stochastic differential equation (SDE).

The paper is organized as follows. In Section 2 we briefly recall Ferguson-Dirichlet process. In Section 3 we consider a different construction of the Brownian motion based on an exchangeable sequence from Dirichlet processes samples which is shown to be a limit of a random walk in Dirichlet random environment. In Section 4, we prove the regularity of the new process and in the Section 5 we give some stochastic calculus and an estima- 
tion of the parameters of DBM.

\section{Ferguson-Dirichlet Process}

Let $(\Omega, \mathcal{B}, \mathcal{P})$ be a fixed probability space. Let $\mathcal{V}$ be a Polish space and let $\mathbf{P}(\mathcal{V})$ denote the set of all probability measures defined on $\mathcal{V}$. The distribution of a random variable, say $Z$, will be denoted by ether $\mathcal{P}_{Z}$ or $\mathcal{L}(Z)$.

The following celebrated random distribution defined by Ferguson [7] plays a central role in our construction. Let $\alpha$ be a finite positive measure on $\mathcal{V}$. A random distribution $P: \Omega \rightarrow \mathbf{P}(\mathcal{V})$ is a Dirichlet process $\mathcal{D}(\alpha)$ if for every $k=2,3, \cdots$ and every measurable partition $B_{1}, \cdots, B_{k}$ of $\mathcal{V}$, the joint distribution of the random vector $\left(P\left(B_{1}\right), \cdots, P\left(B_{k}\right)\right)$ has a Dirichlet distribution with parameters $\left(\alpha\left(B_{1}\right), \cdots, \alpha\left(B_{k}\right)\right)$ Ferguson proved that this definition satisfies the Kolmogorov criteria which yields the existence of such random distributions.

For $c>0$, let $P \operatorname{Dir}(c)$ denote the Poisson-Dirichlet distribution with parameter $c$ (Kingman [8]) which support is the set

$$
\Delta=\left\{q=\left(q_{1}, q_{2}, \cdots, q_{n}, \cdots\right): q_{1} \geq q_{2} \geq \cdots \geq q_{n} \geq \cdots, q_{i} \geq 0, \sum_{i=1}^{\infty} q_{i}=1\right\}
$$

Ferguson has also shown that for a.a. $\omega, P(\omega)$ is a discrete probability measure: there exist an i.i.d. sequence of random variables on $\mathcal{V}$, say $X=\left(X_{i}\right)$, and a sequence of random weights $Q=\left(Q_{i}\right)$ verifying:

$$
\left\{\begin{array}{l}
X_{i} \sim \frac{\alpha}{\alpha(\mathcal{V})} \\
\left(Q_{i}\right) \sim P \operatorname{Pir}(\alpha(\mathcal{V})) \\
Q \perp X
\end{array}\right.
$$

such that

$$
P(\omega)=\sum_{i=1}^{\infty} Q_{i}(\omega) \delta_{X_{i}}(\omega) .
$$

Let $(\Omega, \mathcal{F}, \mathcal{P})$ be a probability space on which are defined all the random variables (r.v.) mentioned in this paper. The probability distribution of a r.v. $X$ will be denoted $\mathcal{P}_{X}$. Equality in distribution is denoted by $\stackrel{d}{=}$.

For any integer $n \geq 2$, let $\Sigma_{n}$ denote the group of permutations of $\{1,2, \cdots, n\}$.

\section{Exchangeable Random Variables}

Definition 1 A sequence $X_{1}, \cdots, X_{n}, \cdots$ of r.v.s is said to be exchangeable if for all $n=2,3, \cdots$

$$
\left(X_{1}, \cdots, X_{n}\right) \stackrel{d}{=}\left(X_{\sigma(1)}, \cdots, X_{\sigma(n)}\right) \text { for all } \sigma \in \Sigma_{n}
$$

Using transpositions, first notice that (2) implies that all the $X_{n}$ have the same distribution, say $\mathcal{L}$ :

$$
X_{i} \stackrel{d}{=} X_{j} \text { for all } i, j \in\{1,2, \cdots\}
$$

and also

$$
\left(X_{i}, X_{j}\right) \stackrel{d}{=}\left(X_{k}, X_{l}\right) \text { for all } i \neq j, k \neq l \in\{1,2, \cdots\} .
$$

The variables $X_{n}$ are assumed to take their values on a separable space $\mathbb{V}$ and $\mathcal{P}(\mathbb{V})$ denote the separable set (for weak convergence topology) of all probability measures defined on $\mathbb{V}$.

An i.i.d. sequence is of course exchangeable but an exchangeable sequence needs neither be independent nor Markov.

For example a sequence $\left(X_{n}\right)$ of centered Gaussian variables with $\mathbb{E}\left(X_{n}^{2}\right)=c>0$ and $\mathbb{E}\left(X_{i} X_{j}\right)=\rho>0$ is exchangeable but not i.i.d. 
Another interesting example of exchangeable sequence is a sample $\left(X_{n}\right)$ from a Dirichlet process $\mathcal{D}\left(c P_{0}\right)$ with precision parameter $c>0$ and mean parameter $P_{0} \in \mathcal{P}(\mathbb{V})$ [7]:

$$
X_{n} \mid P \stackrel{\text { i.i.d. }}{\sim} P, \quad P \sim \operatorname{Dir}(\alpha) .
$$

The following celebrated theorem states that an exchangeable sequence is somewhat conditionally i.i.d. as in the preceding example. It was first established by de Finetti (1931) [9] in the case of Bernoulli variables and by Hewitt-Savage (1955) [10] in the general case. Very elegant proofs can be found in Meyer (1966) [11] p. 191192 and Kingmann (1978) [8].

Theorem 1 (de Finetti-Hewitt-Savage) Let $\left(X_{n}\right)$ be an exchangeable sequence with values in $\mathbb{V}$. Then there exists a probability measure $\mu$ on $\mathcal{P}(\mathbb{V})$ such that

$$
\begin{gathered}
\mathcal{P}\left(X_{1} \in A_{1}, \cdots, X_{n} \in A_{n}\right)=\int_{Q \in \mathcal{P}(\mathbb{V})} Q\left(A_{1}\right) \cdots Q\left(A_{n}\right) \mathrm{d} \mu(Q) \\
\text { If } M(A)=\lim _{n \rightarrow \infty} \frac{1}{n} \sum_{i=1}^{n} \mathbf{1}_{A}\left(X_{i}\right) \text { then } M \sim \mu \\
\mathcal{P}\left(X_{1} \in A_{1}, \cdots, X_{n} \in A_{n} \mid M=Q\right)=Q\left(A_{1}\right) \cdots Q\left(A_{n}\right)
\end{gathered}
$$

In other words, (5) shows that the distribution of an exchangeable sequence is a mixture with mixing measure $\mu$, (6) shows that $\mu$ is the distribution of the weak limit empirical measure and finally (7) shows that if $Q$ is considered as a parameter $\in \mathcal{P}(\mathbb{V})$, then $M$ is a sufficient statistic for estimating $Q$.

Applying (5) with $n=1$ it is seen that the mean $\bar{\mu}$ of $\mu$, defined as $\bar{\mu}(A)=\int_{Q \in \mathcal{P}(\mathbb{V})} Q(A) \mathrm{d} \mu(Q)$, is equal to the common distribution of the $X_{n}$ :

$$
\bar{\mu}=\mathcal{L}
$$

In the example of a sample from the Dirichlet process $\mathcal{D}\left(c P_{0}\right), \mu$ is nothing but the Dirichlet process itself, by definition of such a sample [7], while

$$
\bar{\mu}=\mathcal{L}=P_{0} .
$$

For the rest of the paper it is assumed that $\mathbb{V}=\mathbb{R}$ the real line.

\section{DBM Constructions}

\subsection{DBM Based on Ciesielski Construction}

We follow L. Gallardo [12] pp. 79-80 and 206-208.

Let

$$
h=\mathbf{1}_{\left[0, \frac{1}{2}\right)}-\mathbf{1}_{\left[\frac{1}{2}, 1\right]} .
$$

For any integer $n \geq 1$ and $0 \leq k \leq 2^{n}-1$ let

$$
h_{k, n}=2^{\frac{n}{2}} \mathbf{1}_{\left[\frac{k}{2^{n}} \frac{k+\frac{1}{2}}{2^{n}}\right]}-2^{\frac{n}{2}} \mathbf{1}_{\left[\frac{k+\frac{1}{2}}{2^{n}}, \frac{k+1}{2^{n}}\right)}
$$

that is $h_{k, n}(t)=2^{\frac{n}{2}} h\left(2^{n} t-k\right), \quad t \in[0,1]$.

The functions $1, h$ and $h_{k, n}$ for $n \geq 1$ and $0 \leq k \leq 2^{n}-1$ constitute what is called the Haar Hilbertian basis of $L_{2}([0,1], \mathrm{d} x)$.

Let

$$
s(t)=\int_{0}^{t} h(x) \mathrm{d} x, \quad s_{k, n}(t)=\int_{0}^{t} h_{k, n}(x) \mathrm{d} x .
$$


Note that $s_{k, n}$ is a nonnegative triangle function with support in $\left[\frac{k}{2^{n}}, \frac{k+1}{2^{n}}\right]$ so that

$$
s_{j, n} s_{k, n}=0 \text { if } j \neq k
$$

and

$$
\text { The maximum } 2^{-\frac{n}{2}-1} \text { of } s_{k, n} \text { is reached at } t=\frac{k+\frac{1}{2}}{2^{n}} \text {. }
$$

The functions $t, s$ and $s_{k, n}$ consitute the so called Schauder system.

Now, let $\left(X_{n}\right)=\left(N_{0}, N_{1}, N_{k, n}\right.$ for $n \geq 1$ and $\left.0 \leq k \leq 2^{n}-1\right)$ be a an exchangeable sequence such that $X_{n} \in \mathbb{L}_{2}(\Omega)$ for one (and any) $n$.

Notice that (3) and (4) then imply that

$$
\mathbb{E}\left(X_{n}\right)=m, \quad \mathbb{E}\left(X_{n}^{2}\right)=v^{2}, \quad \mathbb{E}\left(X_{i} X_{j}\right)=c
$$

are constants which do not depend on $n, i$ and $j$.

Let

$$
S_{t}^{(n)}(\omega)=\sum_{k=0}^{2^{n}-1} N_{k, n}(\omega) s_{k, n}(t), \quad \omega \in \Omega, t \in[0,1]
$$

Then

Proposition 2 The series with general term $\sum_{n=1}^{+\infty} S_{t}^{(n)}$ converges in $\mathbb{L}_{2}$ and

$$
X_{t}=t N_{0}+s(t) N_{1}+\sum_{n=1}^{+\infty} S_{t}^{(n)}
$$

defines a stochastic process.

Proof: Due to (10) we have

$$
S_{t}^{(n)}(\omega)^{2}=\sum_{k=0}^{2^{n}-1} N_{k, n}(\omega)^{2} S_{k, n}(t)^{2}
$$

and then (12) applied to the sequence $N_{0}, N_{1}, N_{k, n}$ and (11) give

$$
\mathbb{E}\left(S_{t}^{(n)}(\omega)^{2}\right)=v^{2} \sum_{k=0}^{2^{n}-1} S_{k, n}(t)^{2} \leq v^{2} 2^{-n}
$$

Then $\left\|S_{t}^{(n)}\right\|_{\mathbb{L}_{2}} \leq v 2^{-\frac{n}{2}}$ and $\sum_{n=1}^{+\infty}\left\|S_{t}^{(n)}\right\|_{\mathbb{L}_{2}}<+\infty$ so that $\sum_{n=1}^{+\infty} S_{t}^{(n)}$ converges in $\mathbb{L}_{2}$.

Now, consider the following condition on the tails of $\mathcal{L}$ :

There exists a convergent series with positive general term $c_{n}$ such that the series with general term

$$
2^{n} \mathcal{L}\left(\left(-c_{n} 2^{\frac{n}{2}+1}, c_{n} 2^{\frac{n}{2}+1}\right)^{c}\right)=2^{n} \bar{\mu}\left(\left(-c_{n} 2^{\frac{n}{2}+1}, c_{n} 2^{\frac{n}{2}+1}\right)^{c}\right) \text { converges. }
$$

Proposition 3 If condition (13) holds then a.a. paths of $\left(X_{t}\right)$ are continuous.

Proof: Due to (10) and (11) we have

$$
\left\|S_{t}^{(n)}(\omega)\right\|_{\infty}=\max _{0 \leq k \leq 2^{n}-1} \sup _{t \in\left[\frac{k}{2^{n}}, \frac{k+1}{2^{n}}\right)}\left|N_{k, n}(\omega) s_{k, n}(t)\right|=2^{-\frac{n}{2}-1} \max _{0 \leq k \leq 2^{n}-1}\left|N_{k, n}(\omega)\right|
$$

and (5) implies 


$$
\begin{aligned}
\mathcal{P}\left(\left\|S_{t}^{(n)}\right\|_{\infty}>c_{n}\right) & =\mathcal{P}\left(\max _{0 \leq k \leq 2^{n}-1}\left|N_{k, n}\right|>c_{n} 2^{\frac{n}{2}+1}\right)=1-\mathcal{P}\left(\left|N_{k, n}\right| \leq c_{n} 2^{\frac{n}{2}+1}, 0 \leq k \leq 2^{n}-1\right) \\
& =1-\int_{Q \in \mathcal{P}(\mathbb{V})} Q\left(\left[-c_{n} 2^{\frac{n}{2}+1}, c_{n} 2^{\frac{n}{2}+1}\right]\right)^{2^{n}} \mathrm{~d} \mu(Q) \\
& =\int_{Q \in \mathcal{P}(\mathbb{V})}\left(1-\left(1-Q\left(\left(-c_{n} 2^{\frac{n}{2}+1}, c_{n} 2^{\frac{n}{2}+1}\right)^{c}\right)^{2^{n}}\right)\right) \mathrm{d} \mu(Q) \\
& \leq 2^{n} \int_{Q \in \mathcal{P}(\mathbb{V})}\left(Q\left(\left(-c_{n} 2^{2^{\frac{n}{2}+1}}, c_{n} 2^{\frac{n}{2}+1}\right)^{c}\right)\right) \mathrm{d} \mu(Q) \\
& =2^{n} \bar{\mu}\left(\left(-c_{n} 2^{\frac{n}{2}+1}, c_{n} 2^{\frac{n}{2}+1}\right)^{c}\right)
\end{aligned}
$$

the preceding inequality being due to the inequality $1-(1-q)^{2^{n}} \leq 2^{n} q$ for any $0 \leq q \leq 1$ which is a consequence of finite increments theorem.

Due to (13) we then get that the series with general term $\mathcal{P}\left(\left\|S_{t}^{(n)}\right\|_{\infty}>c_{n}\right)$ converges.

Then by Borel-Cantelli lemma, we have for a.a. $\omega,\left\|S_{t}^{(n)}(\omega)\right\| \leq c_{n}$ for $n$ large enough so that the series $S_{t}^{(n)}(\omega)$ converges uniformly and defines a continuous function of $t$. Thus for a.a. $\omega, X_{t}(\omega)$ is continuous.

As a corollary observe that

Proposition 4 For a sample of $\mathcal{D}(c \mathcal{N}(0,1))$, a.a. paths of $\left(X_{t}\right)$ are continuous.

Proof: Condition (13) holds for $\bar{\mu}=\mathcal{N}(0,1)$ with $c_{n}=\sqrt{2 n} 2^{-\frac{n}{2}-1}$. Indeed, since

$$
a \int_{a}^{+\infty} \mathrm{e}^{-\frac{t^{2}}{2}} \mathrm{~d} t \leq \int_{a}^{+\infty} t \mathrm{e}^{-\frac{t^{2}}{2}} \mathrm{~d} t=\mathrm{e}^{-\frac{a^{2}}{2}}
$$

that is

$$
\int_{a}^{+\infty} \mathrm{e}^{-\frac{t^{2}}{2}} \mathrm{~d} t \leq \frac{\mathrm{e}^{-\frac{a^{2}}{2}}}{a}
$$

holds for any positive number $a$, we have for any $n \geq 1$

$$
\begin{aligned}
2^{n} \mathcal{N}(0,1)\left(\left(-c_{n} 2^{\frac{n}{2}+1}, c_{n} 2^{\frac{n}{2}+1}\right)^{c}\right) & =2^{n} \mathcal{N}(0,1)\left((-\sqrt{2 n}, \sqrt{2 n})^{c}\right)=2^{n+1} \int_{\sqrt{2 n}}^{+\infty} \frac{\mathrm{e}^{-\frac{t^{2}}{2}}}{\sqrt{2 \pi}} \mathrm{d} t \\
& \leq \frac{2^{n+1}}{\sqrt{2 \pi}} \frac{\mathrm{e}^{-n}}{\sqrt{2 n}}=\frac{1}{\sqrt{n \pi}}\left(\frac{2}{\mathrm{e}}\right)^{n} \leq\left(\frac{2}{\mathrm{e}}\right)^{n} .
\end{aligned}
$$

which is the general term of a convergent series.

\subsection{DBM Based on Random Walks}

Let $c>0$ and $\sigma>0$ be fixed.

First, let $\left(Y_{i}\right)$ be a sequence of random variables such that

$$
Y_{i} \mid P \sim P, \quad P \sim \mathcal{i i d}\left(c \mathcal{N}\left(0, \sigma^{2}\right)\right)
$$


which are more explicitly described by the following hierarchical model

$$
\left\{\begin{array}{l}
\left.Y_{i}\right|_{(Q, X)}{ }^{i i d} \sim \sum_{i=1}^{\infty} Q_{i}(\omega) \delta_{X_{i}(\omega)} \\
\left(Q_{i}\right) \sim P \operatorname{Dir}(c) \\
\quad \text { iid } \\
X_{i} \sim \mathcal{N}\left(0, \sigma^{2}\right) \\
Q \perp X
\end{array}\right.
$$

We will rather consider centered variables

$$
\left.U_{i}\right|_{(Q, X)} \stackrel{i i d}{\sim} \sum_{i=1}^{\infty} Q_{i} \delta_{\left[X_{i}-\sum_{j=1}^{\infty} Q_{j} X_{j}\right.} .
$$

Now, consider the following random walk $\left(S_{n}\right)_{n \in \mathbb{N}}$ in Dirichlet random environment, starting from 0:

$$
S_{n}=U_{1}+U_{2}+\cdots+U_{n}
$$

so that we have

$$
\left.S_{n}\right|_{(Q, X)}=\left.\star_{i=1}^{n} U_{i}\right|_{(Q, X)}
$$

It is straightforward that

$$
\sigma(Q, X)=\operatorname{Var}\left(\left.U_{1}\right|_{(Q, X)}\right)=\mathbb{E}\left(\left.U_{1}^{2}\right|_{(Q, X)}\right)=\sum_{i=1}^{\infty} Q_{i}\left[X_{i}-\sum_{j=1}^{\infty} Q_{j} X_{j}\right]^{2}=\sum_{i=1}^{\infty}\left[Q_{i}-Q_{i}^{2}\right] X_{i}^{2}-\sum_{i \neq j}^{\infty} Q_{i} Q_{j} X_{i} X_{j}
$$

Since the $X_{i}$ 's are independent with zero mean, we have

$$
\mathbb{E}\left(\operatorname{Var}\left(\left.U_{1}\right|_{(Q, X)}\right)\right)=\mathbb{E}\left(\sum_{i=1}^{\infty}\left[Q_{i}-Q_{i}^{2}\right]\left(X_{i}\right)^{2}\right) \leq \mathbb{E}\left(\sum_{i=1}^{\infty} Q_{i} X_{i}^{2}\right)=\sigma^{2}
$$

Therefore $\sigma(Q, X)$ is finite a.e. or equivalently, for $\mathcal{P}_{(Q, X)}$ a.a. $(q, x)=\left(\left(q_{i}\right),\left(x_{i}\right)\right) \in \Delta \times \mathbb{R}^{\mathbb{N}^{*}}$

$$
\sigma(q, x)=\sum_{i=1}^{\infty}\left[q_{i}-q_{i}^{2}\right] x_{i}^{2}-\sum_{i \neq j}^{\infty} q_{i} q_{j} x_{i} x_{j}<\infty
$$

For any integer $n \geq 1$ and real number $t \geq 0$ let

$$
S_{t}^{n}=\frac{1}{n^{1 / 2}} S_{[n t]}
$$

where $[x]$ denotes the integer part of $x$.

Let $B^{\sigma}=$ denote a zero mean Brownian motion with variance $\sigma^{2}, B$ denoting the standard Brownian motion.

Proposition 5 For any $0=t_{0}<t_{1}, \cdots,<t_{k}$, we have in the space of distributions

$$
\left.\left(S_{t_{0}}^{n}, S_{t_{1}}^{n}, \cdots, S_{t_{k}}^{n}\right)\right|_{(Q=q, X=x)} \stackrel{d}{\rightarrow} \mathcal{L}\left(B_{t_{0}}^{\sigma(q, x)}, B_{t_{1}}^{\sigma(q, x)}, \cdots, B_{t_{k}}^{\sigma(q, x)}\right) \text { as } n \rightarrow+\infty
$$

where $\sigma(q, x)$ is defined in (15).

\subsection{DBM}

A Brownian motion in Dirichlet random environment (BMDE) is a process $Z$ such that

$$
\left\{\begin{array}{l}
\left.Z\right|_{(Q=q, X=x)}=\mathcal{L}\left(B^{\sigma(q, x)}\right) \\
Q \sim \operatorname{PDir}(c) \\
\quad \text { iid } \\
X_{i} \sim \mathcal{N}\left(0, \sigma^{2}\right)
\end{array}\right.
$$


Proposition 6 If $Z$ is BMDE then its conditional increments are independent Gaussians

$$
Z_{t_{i}}-\left.Z_{t_{i-1}}\right|_{Q=q, X=x}=\mathcal{N}\left(0,\left(t_{i}-t_{i-1}\right) \sigma^{2}(q, x)\right)
$$

The increments $Z_{t_{i}}-Z_{t_{i-1}}$ are orthogonal, are mixtures of Gaussians but need not be independent. Indeed, since

$$
Z_{t_{i+1}}-\left.Z_{t_{i}}\right|_{Q=q, X=x} \sim \mathcal{N}\left(0,\left(t_{i+1}-t_{i}\right) \sigma(q, x)\right)
$$

we see that

$$
Z_{t_{i+1}}-Z_{t_{i}} \sim \int_{\Delta \times \mathbb{R}^{\mathbb{N}^{*}}} \mathcal{N}\left(0,\left(t_{i+1}-t_{i}\right) \sigma(q, x)\right) d \mathcal{P}_{Q, X}(q, x)
$$

\section{Regularity}

Theorem 7 Let $\left(Z_{t}\right)$ be as in (ref) then

$$
\mathbb{E}\left(\left|Z_{t}-Z_{s}\right|^{p}\right) \leq C_{p}|t-s|^{\frac{1}{2}}
$$

so that there exist a continuous version of $\left(Z_{t}\right)$

Proof:

$$
\mathbb{E}\left(\left|Z_{t}-Z_{s}\right|^{p}\right)=\mathbb{E}\left(\mathbb{E}\left(\left|Z_{t}-Z_{s}\right|^{p} \mid Q, X\right)\right)
$$

Since $\mathcal{P}_{Z \mid Q=q, X=x}=\mathcal{P}_{\sigma(q, x) B}$ then

$$
\mathbb{E}\left(\left|Z_{t}-Z_{s}\right|^{p} \mid Q, X\right)=\mathbb{E}\left(\left|B_{t}-B_{s}\right|^{p} \sigma(Q, X)^{p} \mid X, Q\right)=\sigma(Q, X)^{p}|t-s|^{\frac{p}{2}} \mathbb{E}\left(|\mathcal{N}(0,1)|^{p}\right)
$$

where $\sigma(Q, X)=\sum_{i=1}^{\infty} Q_{i}\left[X_{i}-\sum_{j=1}^{\infty} Q_{j} X_{j}\right]^{2}$ Conditional to the $Q,\left[X_{i}-\sum_{j=1}^{\infty} Q_{j} X_{j}\right]$ is a linear combination of $X_{j} \sim \mathcal{N}(0,1)$, then it is a gaussian random variable with 0 mean and variance

$$
\sigma^{2}(Q)=\sum_{i \neq j} Q_{j}+\left(1-Q_{i}\right)^{2} E\left(\int\left(\int\left(\left|Z_{t}-Z_{s}\right|^{p} \mathrm{~d} P_{X \mid Q} \mathrm{~d} P_{Q}\right)\right)\right)
$$

conditional to $Q$.

\section{Simulation and Estimation}

\subsection{Sethuraman Stick-Breaking Construction}

Sethuraman (1994) [13] has shown that the sequence of random distributions

$$
P_{K}(\omega)=\sum_{i=1}^{K} Q_{i}(\omega) \delta_{X_{i}}(\omega)
$$

converges to the Dirichlet process when the random weights $\left(Q_{i}\right)$ are defined by the following stick-breaking construction:

$$
\begin{gathered}
b_{1}, \cdots, b_{K-1} \text { are iid } \operatorname{Beta}(1, c), \quad b_{K}=1 \\
Q_{1}=b_{1}, \quad Q_{k}=\left(1-b_{1}\right) \cdots\left(1-b_{k-1}\right) b_{k}, \quad \text { for any } \quad k=2, \cdots, K .
\end{gathered}
$$

\subsection{Simulation Algorithm}

A path of the $\operatorname{BMDE}(c, \sigma)$ process $\left(Z_{0}=0, Z_{t_{1}}, \cdots, Z_{t_{n}}\right)$ can be simulated as follows:

Let $\mathrm{d} t=t_{i+1}-t_{i}>0$ be small enough and let $K$ be the stick-breaking precision

Draw $q=\left(q_{1}, q_{2}, \cdots, q_{K}\right)$ from (19) 
Draw $x=\left(x_{1}, q_{2}, \cdots, x_{K}\right)$ with $x_{i}^{\prime} s \sim \mathcal{N}\left(0, \sigma^{2}\right)$

Compute $\sigma_{K}^{2}(q, x)$ by truncating (15)

Put $Z_{0}=0$ and draw $n$ points $Z_{t_{i}}$ such that $Z_{t_{i+1}}-Z_{t_{i}}{ }_{i i d} \sim \mathcal{N}\left(0, \mathrm{~d} t \sigma_{K}^{2}(q, x)\right)$

\subsection{Estimation}

Using proposition 6 we can show that

$$
\mathbb{E}\left[\left(Z_{t_{i+1}}-Z_{t_{i}}\right)^{2}\right]=\left(t_{i+1}-t_{i}\right) \sigma\left[1-\frac{c}{(c+2)(c+1)}\right]
$$

\section{Stochastic Calculus}

Consider $\left(\mathcal{F}_{t}\right)_{t \geq 0}$ the natural filtration defined by $Z$, that is $\mathcal{F}_{t}=\sigma\left(Z_{s}, s \leq t\right)$ the sigma algebra generated by $\left\{Z_{s}, s \leq t\right\}$ A random process $(f(t))_{t \geq 0}$ is a step process if there exist a finite sequence of numbers $0=t_{0}<t_{1}<\cdots<t_{n}$ and square integrable random variables $\eta_{0}, \eta_{1}, \cdots, \eta_{n-1}$ such that

$$
f(t)=\sum_{i=1}^{n-1} \eta_{j} I_{\left[t_{t_{j}}, t_{j+1}\right)}(t)
$$

where $\eta_{j}$ is $\mathcal{F}_{t_{j}}$-measurable for $j=0, \cdots, n-1$ The set of random step processes will be denoted by $\mathcal{M}_{\text {step }}$ Observe that the assumption that the $\eta_{j}$ are to be $\mathcal{F}_{t}$-measurable ensures that $f(t)$ is adapted to the filtration $\mathcal{F}_{t}$. The assumption that the $\eta_{j}$ are square integrable ensures that $f(t)$ is square integrable for each $t$. The stochastic integral of $f \in \mathcal{M}_{\text {step }}$ is defined as

$$
I(f)=\sum_{i=1}^{\infty} \eta_{i}\left(Z_{t_{i+1}}-Z_{t_{i}}\right)
$$

Proposition 8 For $f \in \mathcal{M}_{\text {step }}$, we have $I(f) \in L_{2}$ and

$$
\mathbb{E}\left(|I(f)|^{2}\right)=K(c, \sigma) \mathbb{E}\left(\int_{0}^{\infty}|f(t)|^{2} \mathrm{~d} t\right)
$$

where $K(c, \sigma)=\sigma\left[1-\frac{c}{(c+2)(c+1)}\right]$

This enables us to define with standard techniques, the stochastic integral

$$
\int_{0}^{t} f\left(Z_{s}\right) \mathrm{d} Z_{s}
$$

for any continuous function $f$.

Proposition 9 The stochastic process $\left(\int_{0}^{t} f^{\prime}\left(Z_{s}\right) \mathrm{d} Z_{s}\right)_{t \geq 0}$ is a $\mathcal{F}_{t}^{\prime}$-martingale

\section{Proof:}

Let $t$ and $s$ two reals numbers such that $s \leq t$, let $k \in \mathbb{N}$ such that $0=t_{0}<t_{1}<\cdots<t_{k} \leq s \leq t_{k+1}<t_{n}$, let $\eta_{j}=f\left(Z_{\xi_{j}}\right)$

$$
\begin{aligned}
\left.\mathbb{E}\left(\int_{0}^{t} f^{\prime}\left(Z_{s}\right) \mathrm{d} Z_{s}\right)\right|_{\mathcal{F}_{s}^{\prime}} & =\mathbb{E}\left(\left.\lim _{n \rightarrow \infty} \sum_{i=1}^{n} \eta_{j} \Delta_{j} Z\right|_{\mathcal{F}_{s}^{\prime}}\right)=\lim _{n \rightarrow \infty} \mathbb{E}\left(\sum_{i=1}^{k} \eta_{j} \Delta_{j} Z+\left.\sum_{i=k+1}^{n} \eta_{j} \Delta_{j} Z\right|_{\mathcal{F}_{s}^{\prime}}\right) \\
& =\lim _{n \rightarrow \infty} \mathbb{E}\left(\left.\sum_{j=1}^{k} \eta_{j} \Delta_{j} Z\right|_{\mathcal{F}_{s}^{\prime}}\right)+\mathbb{E}\left(\left.\sum_{j=k+1}^{n} \eta_{j} \Delta_{j} Z\right|_{\mathcal{F}_{s}^{\prime}}\right) .
\end{aligned}
$$

where

since for every $j \in\{0, \cdots, k\}, \quad \eta_{j}$ and $\Delta_{j} Z \mid(p, x)$ is $\mathcal{F}^{\prime}$-measurable then, 


$$
\mathbb{E}\left(\left.\eta_{j} \Delta_{j} Z\right|_{\mathcal{F}_{s}^{\prime}}\right)=\eta_{j} \mathbb{E}\left(\left.\Delta_{j} Z\right|_{\mathcal{F}_{s}^{\prime}}\right)=\eta_{j} \Delta_{j} Z
$$

On the other hand for every $j \in\{k+1, \cdots, n\}$ using the zero means of increment $\Delta_{j} Z$ conditional to $(p, x)$.

$$
\begin{aligned}
\mathbb{E}\left(\left.\Delta_{j} Z\right|_{\mathcal{F}_{s}^{\prime}}\right) & =a_{j} \psi((p, x))+b_{j} \varphi\left(B_{j}, B_{j+1}\right) \\
& =\psi(p, x)\left\langle\psi(p, x), \Delta_{j} Z\right\rangle+\varphi\left(B_{j}, B_{j+1}\right)\left\langle\varphi\left(B_{j}, B_{j+1}\right), \Delta_{j} Z\right\rangle \\
& =\psi(p, x) \int\left[\int y \mathrm{~d} P_{\Delta_{j} Z^{(p, x)}}(y)\right] \psi(p, x) \mathrm{d} P(p, x) \\
& =\varphi\left(B_{j}, B_{j+1}\right)\left[\int \varphi\left(B_{j}, B_{j+1}\right) \mathrm{d} P\left(B_{j}, B_{j+1}\right)\right]\left[\int\left(\int y \mathrm{~d} P_{\Delta_{j} Z^{(p, x)}}(x) P(p, x)\right)\right] \\
& =0
\end{aligned}
$$

consequently,

$$
\mathbb{E}\left(\left.\int_{0}^{t} f^{\prime}\left(Z_{s}\right) \mathrm{d} Z_{s}\right|_{\mathcal{F}_{s}^{\prime}}\right)=\int_{0}^{s} f^{\prime}\left(Z_{l}\right) \mathrm{d} Z_{l}
$$

\section{Itô Formulae}

In this paragraph we shall give an expression of Itô formulae of the process $Z$

Proposition 10

$$
\left.\int_{(q, x) \in \Delta \times \mathbb{R}^{\mathbb{N}^{*}}} \int_{0}^{t} f\left(\left.Z_{s}\right|_{(q, x)}\right) \mathrm{d} Z_{s}\right|_{(p, x)} \mathrm{d} P(p, x)=\int_{0}^{t} f\left(Z_{s}\right) \mathrm{d} Z_{s}
$$

Proof:

Since

$$
\mathbb{E}\left(\lim _{n \rightarrow \infty} \mathbb{E}\left(\left|\sum_{i=1}^{n-1} \eta_{i}\left(\left.Z_{t_{i+1}}\right|_{(p, x)}-\left.Z_{t_{i}}\right|_{(p, x)}\right)\right|^{2}\right)\right)=\int \lim _{n \rightarrow \infty} \sum_{i=1}^{n-1} \mathbb{E}\left(\eta_{i}^{2}\right) \mathbb{E}\left(\left[\left.Z_{t_{i+1}}\right|_{(p, x)}-\left.Z_{t_{i}}\right|_{(p, x)}\right]^{2}\right)
$$

Suppose that

$$
X_{n}(p, x)(.)=\mathbb{E}\left[\left|\sum_{i=1}^{n-1} \eta_{i}\left(\left.Z_{t_{i+1}}(p, x)\right|_{(p, x)}-\left.Z_{t_{i}}(p, x)\right|_{(p, x)}\right)\right|^{2}\right]=\sum_{i=1}^{n-1} \mathbb{E}\left(\eta_{i}^{2}\right) \mathbb{E}\left(\left[\left.Z_{t_{i+1}}\right|_{(p, x)}-\left.Z_{t_{i}}\right|_{(p, x)}\right]^{2}\right)
$$

For almost surely $\omega^{\prime}$,

$$
\lim _{n \rightarrow \infty} X_{n}(p, x)=\lim _{n \rightarrow \infty} \sum_{i=1}^{n-1} \mathbb{E}\left(\eta_{i}^{2}\right) \mathbb{E}\left(\left.Z_{t_{i+1}}\right|_{(p, x)}-\left.Z_{t_{i}}\right|_{(p, x)}\right)^{2}=\Delta_{j} t \sigma(p, x) \mathbb{E}\left(\int_{0}^{\infty}|f(t)|^{2} \mathrm{~d} t\right)
$$

On the other hand for almost surely $\omega^{\prime}$ and for any $n \in \mathbb{N}$

$$
\left|X_{n}(p, x)\right| \leq \Delta_{j} t \sigma(p, x) \mathbb{E}\left(\int_{0}^{\infty}|f(t)|^{2} \mathrm{~d} t\right) \in L^{1}(P)
$$

Therefore according to the dominus convergence theorem,

$$
\begin{aligned}
\int \lim _{n \rightarrow \infty} \mathbb{E}\left(\left|\sum_{i=1}^{n-1} \eta_{i}\left(\left.Z_{t_{i+1}}\right|_{(p, x)}-\left.Z_{t_{i}}\right|_{(p, x)}\right)\right|^{2}\right) \mathrm{d} P(p, x) & =\lim _{n \rightarrow \infty} \int \mathbb{E}\left(\left|\sum_{i=1}^{n-1} \eta_{i}\left(\left.Z_{t_{i+1}}\right|_{(p, x)}-\left.Z_{t_{i}}\right|_{(p, x)}\right)\right|^{2}\right) \mathrm{d} P(p, x) \\
& =\lim _{n \rightarrow \infty} \int \sum_{i=1}^{n-1} \mathbb{E}\left(\eta_{i}^{2}\right)\left(\left.Z_{t_{i+1}}\right|_{(p, x)}-\left.Z_{t_{i}}\right|_{(p, x)}\right)^{2} \\
& =\int \sigma(p, x) \mathbb{E}\left(\int_{0}^{\infty}|f(t)|^{2} \mathrm{~d} t\right) \mathrm{d} P(p, x) \\
& =K(c, \sigma) \mathbb{E}\left(\int_{0}^{\infty}|f(t)|^{2} \mathrm{~d} t\right)
\end{aligned}
$$


this means that

$$
\left.\iint_{0}^{t} f\left(\left.Z_{s}\right|_{(p, x)}\right) \mathrm{d} Z_{s}\right|_{(p, x)} \mathrm{d} P(p, x)=\int_{0}^{t} f\left(Z_{s}\right) \mathrm{d} Z_{s}
$$

as required.

Proposition 11 Let $f$ be a bounded and 2 times derivable function, then

$$
\int f\left(Z_{t}\right) \mathrm{d} P(p, x)=\int f\left(Z_{0}\right) \mathrm{d} P(p, x)+\int_{0}^{t} f^{\prime}\left(Z_{s}\right) \mathrm{d} Z_{s}+\frac{1}{2} \int_{0}^{t} \int f^{\prime \prime}\left(Z_{s}\right) \mathrm{d} P(p, x) \mathrm{d} s
$$

\section{Conclusion}

We have extended Brownian motion in dirichlet random environment for the application on the Dirichlet hierarchical models in order to deal with temporal data such as solutions of SDE with stochastic drift and volatility. It can be thought that the process on which are based these parameters belongs to a certain well-known class of processes, such as continuous time Markov chains. Then, we think that a Dirichlet prior can be put on the path space, that is a functional space. It seems to us that the estimation procedure in such a context is an interesting topic for future works.

\section{References}

[1] Dahl, B.D. (2006) Model-Based Clustering for Expression Data via a Dirichlet Process Mixture Model. In: Do, K.-A., Meller, P. and Vannucci, M., Eds., Bayesian Inference for Gene Expression and Proteomics, Cambridge University Press, Cambridge.

[2] Ishwaran, H. and James, L.F. (2002) Approximate Dirichlet Processes Computing in Finite Normal Mixtures: Smoothing and Prior Information. Journal of Computational and Graphical Statistics, 11, 209-230. http://dx.doi.org/10.1198/106186002411

[3] Blei, M.D. and Ng, Y.A. and Jordan, I.M. (2003) Latent Dirichlet Allocation. Journal of Machine Learning Research, 3, 993-1022.

[4] Bertrand, P. (1996) Estimation of the Stochastic Volatility of a Diffusion Process I. Comparison of Haar Basis Estimator and Kernel Estimators. INRIA Rocquencourt Technical Report, 2739.

[5] Deshhpande, A. and Ghosh, M.K. (2007) Risk Minimizing Option Pricing in a Regime Switching Market (to appear).

[6] Faires, H. (2012) SDEs in Dirichlet Random Environment IJSS 1, 5566.

[7] Ferguson, T.S. (1973) A Bayesian Analysis of Some Nonparametric Problems. Annals of Statistics, 1, 209-230. http://dx.doi.org/10.1214/aos/1176342360

[8] Kingman, J.F.C. (1978) Uses of exchangeability. Annals of Probability, 6, 183197. http://dx.doi.org/10.1214/aop/1176995566

[9] de Finetti, B. (1931) Funzione caratteristica di un fenomeno aleatorio. Attidella R. Academia Nazionale dei Lincei, Serie 6. Memorie, Classe di Scienze Fisiche, Mathematice e Naturale, 4, 251299.

[10] Hewitt, E. and Savage, L.J. (1955) Symmetric Measures on Cartesian Products. Transactions of the American Mathematical Society, 80, 470501. http://dx.doi.org/10.1090/S0002-9947-1955-0076206-8

[11] Meyer, P.-A. (1966) Probabilités et potentiel. Hermann, Paris.

[12] Gallardo, L. (2008) Mouvement Brownien et calcul d’Itô. Hermann, Paris.

[13] Sethuraman, J. (1994) A Constructive Definition of Dirichlet Priors. Statistica Sinica, 4, 639-650. 
Scientific Research Publishing (SCIRP) is one of the largest Open Access journal publishers. It is currently publishing more than 200 open access, online, peer-reviewed journals covering a wide range of academic disciplines. SCIRP serves the worldwide academic communities and contributes to the progress and application of science with its publication.

Other selected journals from SCIRP are listed as below. Submit your manuscript to us via either submit@scirp.org or Online Submission Portal.
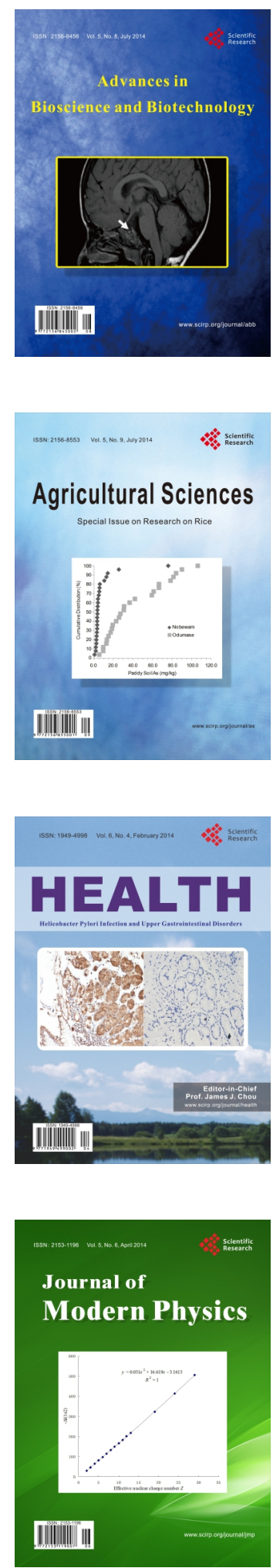
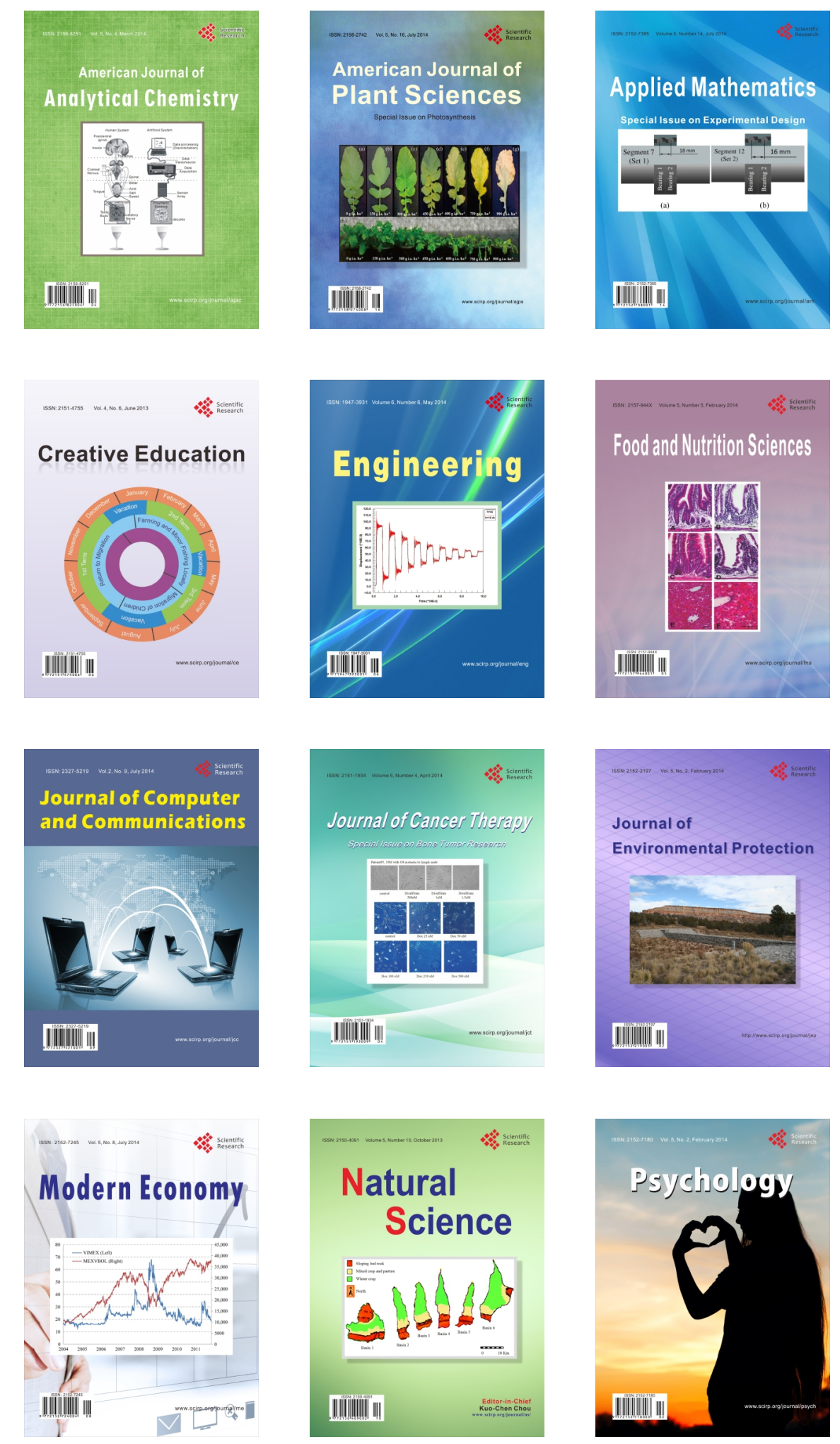